LA-UR-01-0847

Approved for public release; distribution is unlimited.

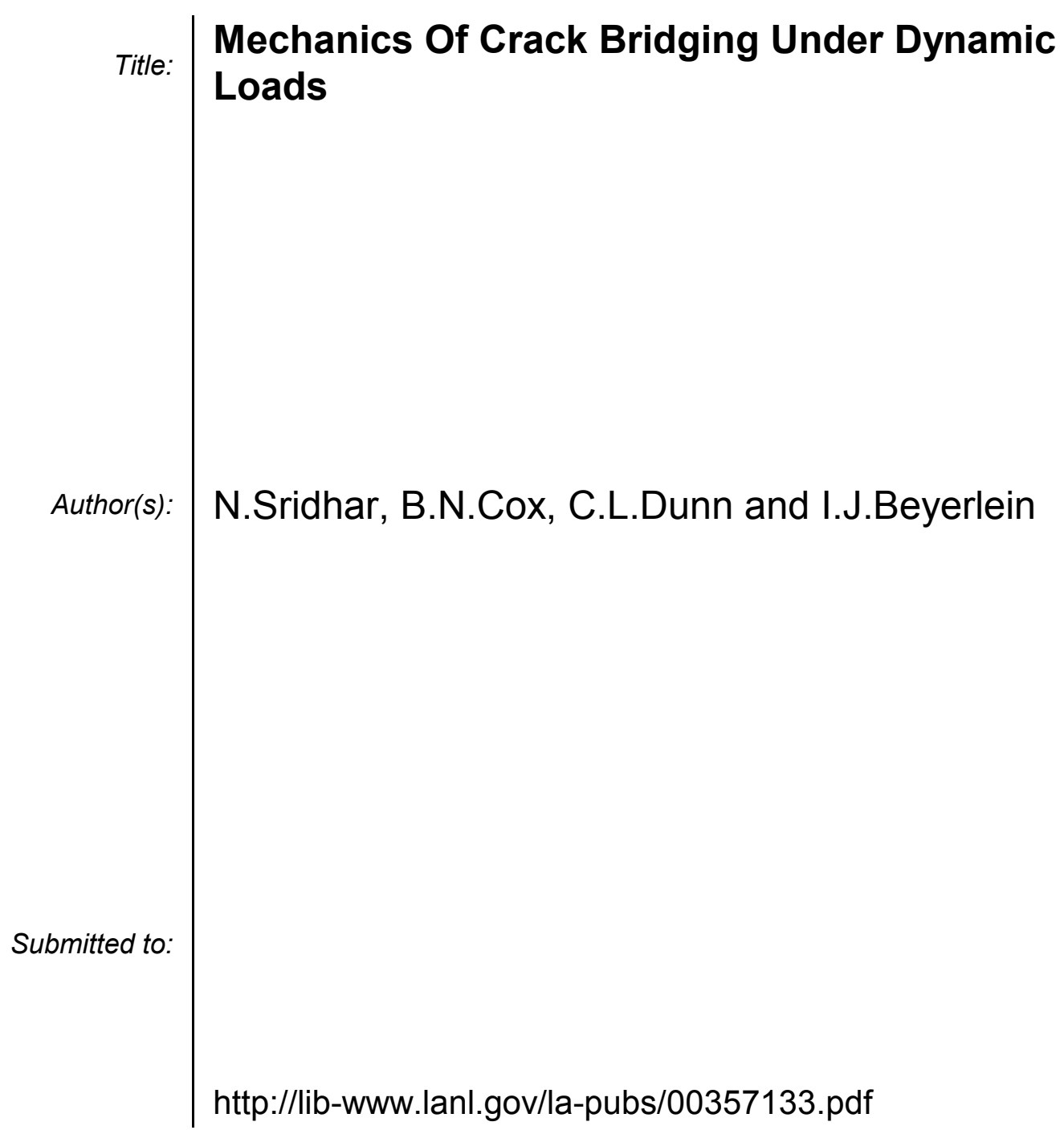

Los Alamos National Laboratory, an affirmative action/equal opportunity employer, is operated by the University of California for the U.S. Department of Energy under contract W-7405-ENG-36. By acceptance of this article, the publisher recognizes that the U.S. Government retains a nonexclusive, royaltyfree license to publish or reproduce the published form of this contribution, or to allow others to do so, for U.S. Government purposes. Los Alamos National Laboratory requests that the publisher identify this article as work performed under the auspices of the U.S. Department of Energy. Los Alamos National Laboratory strongly supports academic freedom and a researcher's right to publish; as an institution, however, the Laboratory does not endorse the viewpoint of a publication or guarantee its technical correctness. 


\title{
Mechanics Of Crack Bridging Under Dynamic Loads
}

\author{
N.Sridhar ${ }^{1 *}$, B.N.Cox ${ }^{1}$, C.L.Dunn ${ }^{2}$ and I.J.Beyerlein ${ }^{3}$ \\ ${ }^{1}$ Rockwell Science Center, 1049 Camino Dos Rios, Thousand Oaks, CA, U.S.A \\ ${ }^{2}$ Massachusetts Institute of Technology, Cambridge, MA, U.S.A \\ ${ }^{3}$ Los Alamos National Laboratory, Los Alamos, NM, U.S.A
}

\begin{abstract}
A bridging law for fiber reinforced composites under dynamic crack propagation conditions has been derived. Inertial effects in the mechanism of fibre pullout during dynamic propagation of a bridged crack are critically examined for the first time. By reposing simple shear lag models of pullout as problems of dynamic wave propagation, the effect of the frictional coupling between the fibres and the matrix is accounted for in a fairly straightforward way. The solutions yield the time-dependent relationship between the crack opening displacement and the bridging traction. Engineering criteria and the role of material and geometrical parameters for significant inertial effects are identified.
\end{abstract}

\section{INTRODUCTION}

The mechanics of pullout and the resulting traction law have been much studied and are well understood for static loading. Simple analytical forms are available for $p(u)$ when the frictional coupling of the reinforcement to the matrix is uniform and slip extends over distances that are large compared to the reinforcement diameter [1-3]. In this limit, which is a common case in ceramic composites and textile polymeric composites, the shear lag model of load transfer between the reinforcement and the matrix is accurate.

In this paper, we extend existing models of the mechanics of pullout to high loading rates. An approach to evaluating a traction law that takes account of the inertia of the reinforcement and the matrix is formulated as a direct extension of the elementary static loading model. Attention will be focused on identifying a characteristic time for the frictional pullout problem that will allow rapid assessment of when inertial effects will be important.

\section{THEORY}

The crack propagation and pullout problem can idealized as follows. A matrix crack propagates on the plane $z=0$ and is bridged by intact fibres in its wake. The crack propagation problem is represented by replacing the process zone by elastic composite material down to the fracture plane and representing the phenomena within the process zone by bridging tractions applied continuously on the fracture surfaces. The bridging tractions, $p$, are related to the axial stress in the fibres. The total crack opening displacement, $2 u$, is defined as the difference in the actual displacement evaluated across the process zone and the displacement that would be 
expected if the material in the process zone was elastic. The traction law, $p(u)$, is derived by considering the micromechanics of frictional sliding. The traction boundary conditions are as follows. At $z=0$, the matrix is traction-free, while the fibres sustain the axial traction $T$. At $z=$ $l_{\mathrm{s}}$, the strain in the fibres and the matrix must equal the average strain in the composite adjacent to the process zone $\left(z>l_{\mathrm{s}}\right)$. Shear tractions may arise along the vertical boundaries of the representative volume (parallel to $z$ ), but these are neglected.

\section{Micromechanics of Dynamic Pullout}

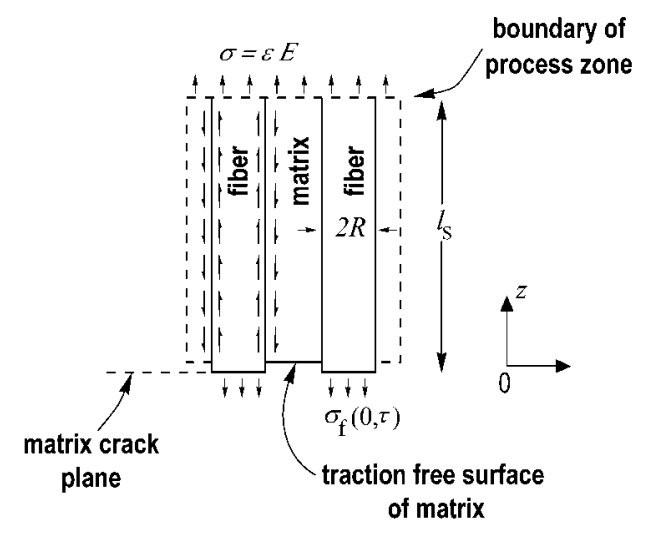

Figure 1: Schematic of the dynamic pullout problem in a composite near the fracture plane.

When friction is the only active force of resistance, the dynamic pullout problem is one of wave propagation along a fibre in the presence of frictional retardation. A representative volume (Fig. 1) consists of cylindrical fibres of radius $R$ and volume fraction $f$ embedded in a matrix $(z \geq$ $0)$. The fibre and the matrix have axial Young's moduli $E_{\mathrm{f}}$ and $E_{\mathrm{m}}$ and densities $\rho_{\mathrm{f}}$ and $\rho_{\mathrm{m}}$ respectively. The axial displacement, strain, and stress of the fibre and the matrix are denoted $u_{\mathrm{f}}$ and $u_{\mathrm{m}}, \varepsilon_{\mathrm{f}}$ and $\varepsilon_{\mathrm{m}}$, and $\sigma_{\mathrm{f}}$ and $\sigma_{\mathrm{m}}$, respectively. The axial displacements will be assumed to be the only nonzero displacement components induced by loading and to be uniform across any section of the fibre or the matrix. Thus the displacement, strain, and stress in the fibre and the matrix are functions of $z$ and $t$ only. The fibres are coupled to the matrix by friction tractions, $\tau_{\mathrm{f}}$, which are assigned the following properties. If relative motion exists between the fibres and the matrix, then, $\tau_{\mathrm{f}}=\tau$ if $\dot{u}_{\mathrm{f}}<\dot{u}_{\mathrm{m}}$ and $\tau_{\mathrm{f}}=-\tau$ if $\dot{u}_{\mathrm{f}}>\dot{u}_{\mathrm{m}}$. The dot indicates time differentiation, $\tau$ is a positive constant and $\tau_{\mathrm{f}}>0$ indicates friction tractions acting on the fibres in the positive $\mathrm{z}$ direction. With such a friction law, the dynamic wave equations for the fibre and the matrix are:

$$
\begin{aligned}
& \frac{\partial^{2} u_{\mathrm{f}}}{\partial z^{2}}=-\frac{2 \theta \tau}{R E_{\mathrm{f}}}+\frac{1}{c_{\mathrm{f}}^{2}} \frac{\partial^{2} u_{\mathrm{f}}}{\partial t^{2}} \\
& \frac{\partial^{2} u_{\mathrm{m}}}{\partial z^{2}}=\frac{2 f}{1-f} \frac{\theta \tau}{R E_{\mathrm{m}}}+\frac{1}{c_{\mathrm{m}}^{2}} \frac{\partial^{2} u_{\mathrm{m}}}{\partial t^{2}}
\end{aligned}
$$

where $c_{i}=\sqrt{E_{i} / \rho_{i}}, \theta=1$ if $\dot{u}_{\mathrm{m}}>\dot{u}_{\mathrm{f}}$ and $\theta=-1$ otherwise; The boundary conditions are as follows: At the fracture plane $u_{\mathrm{f}}(0, t)=0$ and $\sigma_{\mathrm{m}}(0, t)=0$; At the boundary of the process zone $\left(z=l_{\mathrm{s}}\right), u_{\mathrm{f}}=u_{\mathrm{m}}=u_{\mathrm{c}}$, where $u_{\mathrm{c}}$ is the displacement of the adjacent intact composite. Conditions also exist on stress or strain and particle velocities at the process zone boundary. These 
conditions depend on the nature of the loading history, which can be expressed as the function $\varepsilon(t)$, where $\varepsilon$ is the strain in the $z$ direction in the intact composite adjacent to the process zone boundary.

In the depiction of Fig. 1, the process zone boundary will propagate away from the fracture plane as $\varepsilon(t)$ rises. We will first consider $\varepsilon(t)$ to rise continuously and monotonically from zero. The location of the zone boundary at time $t$ may then be written as $l_{\mathrm{s}}=\eta(t) c_{\mathrm{m}} t$, where the function $\eta(t)$ depends on material and geometrical parameters and the form of $\varepsilon(t)$ and it will be shown to be bounded by $0<\eta(t) \leq 1$. For the loading conditions considered, the additional boundary conditions at $z=l_{\mathrm{s}}$ are: $\dot{u}_{\mathrm{f}}=\dot{u}_{\mathrm{m}}=\dot{u}_{\mathrm{c}}$ and $\varepsilon_{\mathrm{f}}=\varepsilon_{\mathrm{m}}=\varepsilon(t)$. If the load history, $\varepsilon(t)$, possesses discontinuities, e.g., a step load, then discontinuities in stress and velocity will also propagate at the boundary of the process zone [4].

\section{Composite Stress Rising Linearly in Time}

A case of representative interest for dynamic bridged crack problems and for which analytical results can be found is that of a load or bridging traction that increases linearly in time. A linearly increasing load might give insight, for example, into bridging effects in a specimen in which substantial bending arises, such as a standard double cantilever beam delamination specimen. In such specimens, the crack profile is often approximately linear and the rate of increase of the bridging tractions at any point might also be approximately linear if the crack propagates at approximately constant speed. Let $\varepsilon(t)=k t$, where $k$ is constant and all displacements and boundary tractions are zero for $t<0$. In this case, $\theta=1$ and the wave equations have the solutions

$$
\begin{aligned}
& u_{\mathrm{f}}=-\frac{k}{c_{\mathrm{m}}} \alpha z^{2}+[1+2 \eta \alpha] \varepsilon z \\
& u_{\mathrm{m}}=\frac{k}{c_{\mathrm{m}}} \frac{1}{2 \eta} z^{2}+\frac{1}{2}[1+2 \eta \alpha] \varepsilon l_{\mathrm{s}}
\end{aligned}
$$

where $\eta$ is independent of time and satisfies

$$
\alpha \eta^{3}+1 / 2 \eta^{2}+\beta \alpha \eta-1 / 2=0
$$

with the dimensionless parameters $\alpha$ and $\beta$ given by

$$
\alpha=\frac{\tau c_{\mathrm{m}}}{E_{\mathrm{f}} R k} \quad \& \quad \beta=\frac{f}{1-f} \frac{E_{\mathrm{f}}}{E_{\mathrm{m}}}
$$

Analysis shows that Eqn. (3) has only one real root, which always lies in $(0,1)$. The displacement, $u$, is given by the common fibre/matrix displacement at the boundary of the process zone minus the displacement expected if the process zone material were elastic:

$$
u=\frac{c_{m} \alpha \eta^{2}}{k E^{2}} p^{2}
$$

Equation 4 constitutes the traction law for the case of linearly rising loads. The limit of very fast loading corresponds to $k \rightarrow \infty$, whereupon $\alpha \rightarrow 0$ and $\eta \rightarrow 1$, since the first and third terms in Eqn. (2c) become negligible. The disturbance then propagates at the bar wave speed in the matrix. Static loading is represented by the limit $\alpha \rightarrow \infty$, for which the first two terms of Eqn. (2c) become small and one has the asymptotic solution $\eta \rightarrow 1 /(2 \alpha \beta)$ which coincides with the 
results obtained by McCartney [2]. We can also show that for loads that increase linearly with time [5], the form of the traction law is identical in the static and dynamic cases and

$$
\frac{u}{u^{(\mathrm{st})}}=2 \alpha \beta \eta
$$

Since $2 \alpha \beta \eta<1$ always, inertial effects increase the stiffness, $d p / d u$, of the traction law for loads that increase linearly in time.

\section{Fibre Pullout from a Rigid Matrix}

In this section, we examine pullout of a single fibre from a rigid matrix to which it is coupled by friction. The problem considered for a rigid matrix is the case of the fibre being loaded on the fracture plane by tractions, $T(t)$. The response of the system that is of interest is wholly represented by the load point displacement, i.e., the displacement, $u_{0}(t)$, of the fibre at $z=0$, since the matrix is rigid (motionless). Specifying a boundary condition on the fracture plane, rather than at the end of the slip zone, as for the composite problem, is preferred because it allows for a simpler statement on the imposed loading. This is then exactly identical to the problem of an end loaded rod damped by friction [6]. Analytical solutions can be found for step loads as well as for linearly increasing loads.

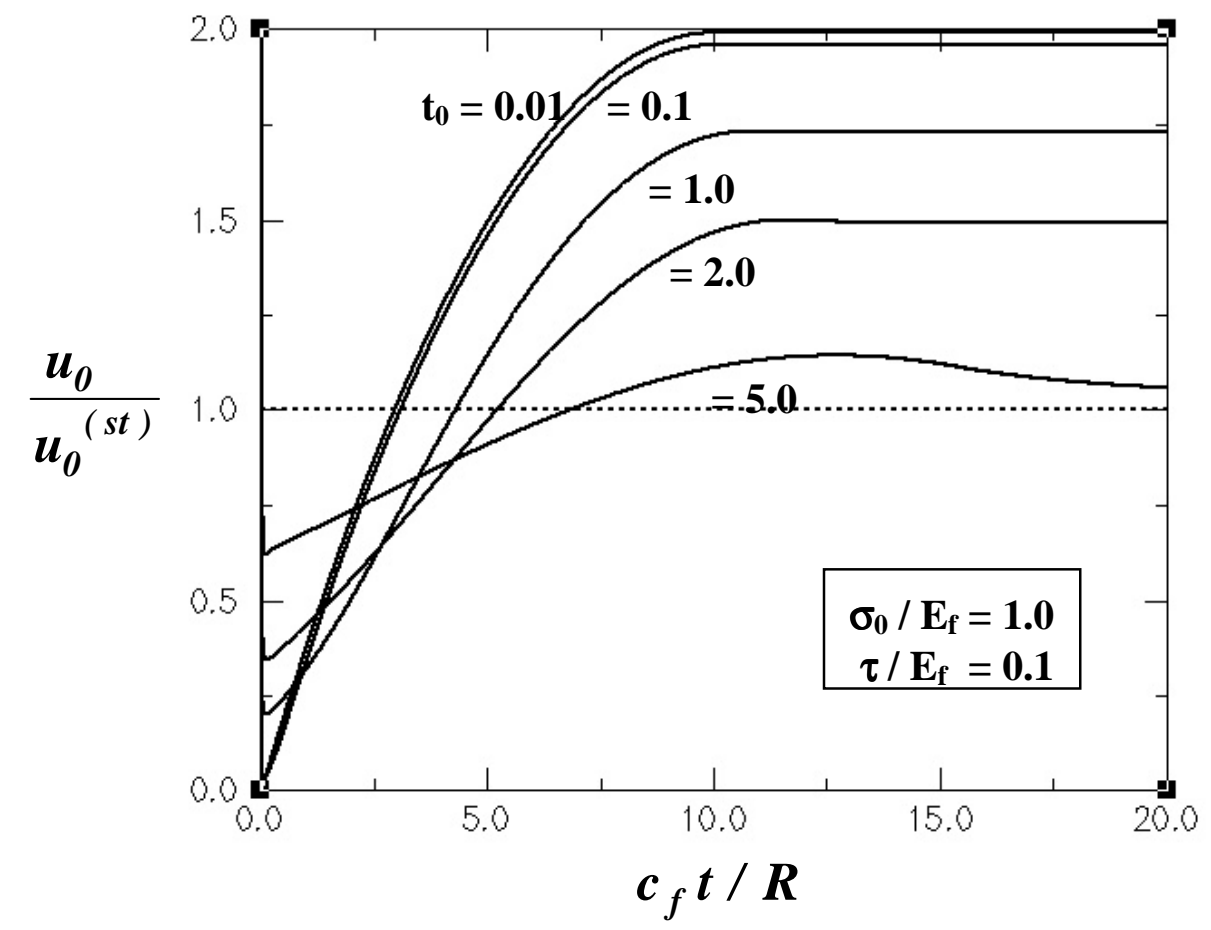

Figure 2: The variation of the load point displacement (normalized by the corresponding quasistatic value) as a function of the normalized time for various values of $t_{0}$.

The most interesting features are the following. While the load point displacement is reduced by inertial effects for linearly increasing loads, for a step load it is increased. For a linearly increasing load, $\varepsilon_{0}=k t$, 


$$
\frac{u_{0}}{u_{0}^{(s t)}}=2\left[\frac{\sqrt{1+\left(k t_{r}\right)^{2}}-1}{\left(k t_{r}\right)^{2}}\right]
$$

where the characteristic time of the system, $t_{\mathrm{r}}$, is given by $t_{r}=E_{f} R /\left(\tau_{c_{f}}\right)$, with the subscript "r" referring to the matrix being rigid. The expression in (6) is always less than unity. In contrast, for a step function load $\varepsilon_{0}(t)=\varepsilon_{0}$, a constant for $t>0$, all motion ceases when $t=\varepsilon_{0} t_{\mathrm{r}}$, at which point $u_{0}=2 u_{0}^{\text {(st) }}$ : inertial effects double the displacement expected from loading statically to the same applied load. Correspondingly, the strain gradient left in the fibre following dynamic (step) loading is exactly half that found after static loading.

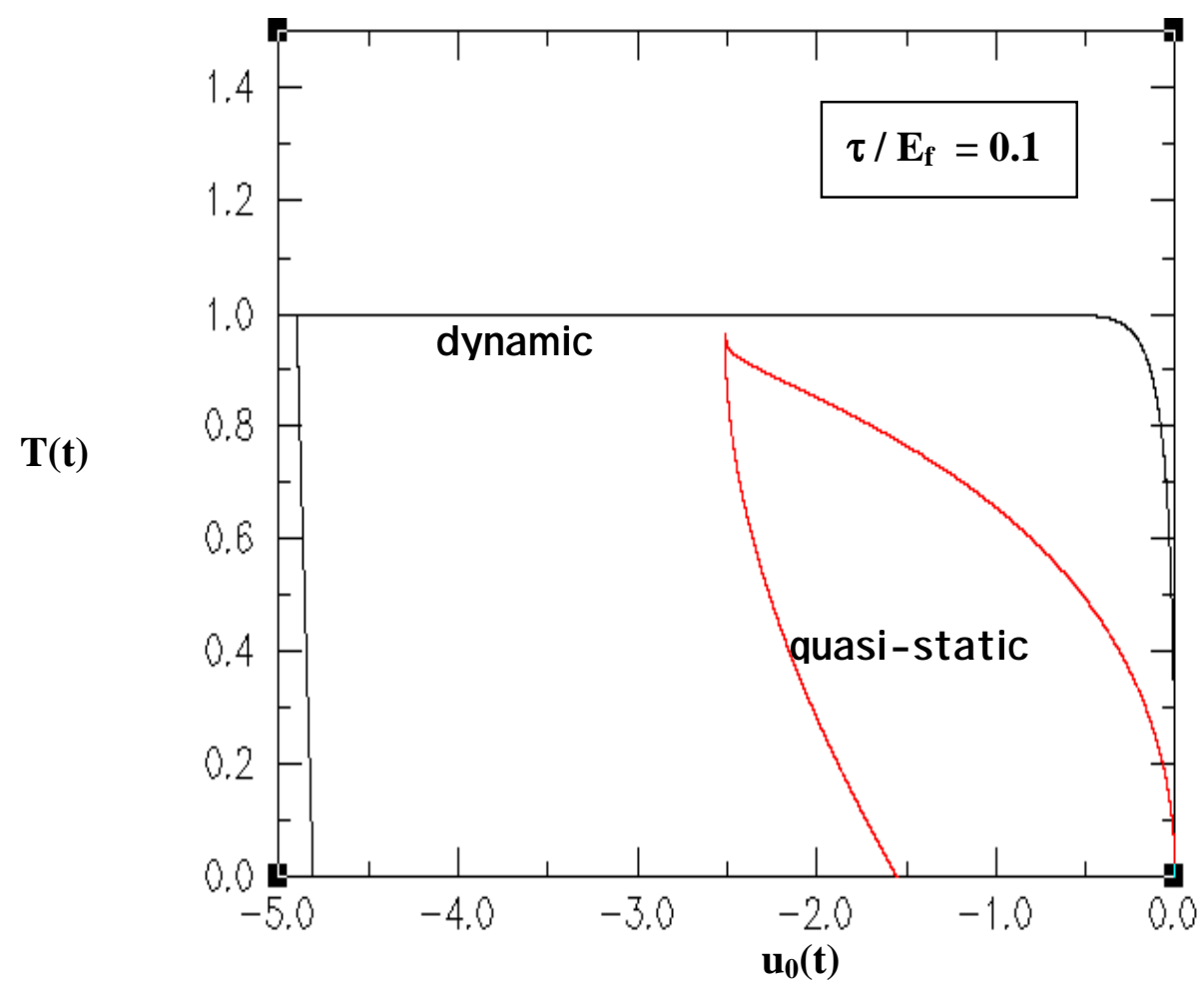

Figure 3: The traction law for load-unload case. The traction is normalized by the fibre modulus and the load point displacement is normalized by the fibre radius.

However, for more general loading configurations, a finite difference numerical scheme is used to solve the non-linear wave equation [7]. The finite difference method is based on a second-order upwind scheme written for the linearized scalar form of the Euler equations. Further, by directional discretization of the derivatives, the local Euler equation is solved for discontinuous neighboring states [8]. This approach results in artificial viscosity being introduced into the governing equation but solutions thus generated allow resolution of the discontinuities without oscillations. This last aspect is important since discontinuities in stresses and particle velocities at the wavefront is an essential phenomena for loading histories having discontinuous time derivatives. 
With the aid of the numerical method, we compute the load-point displacement when the boundary traction is $T(t)=\sigma_{0}\left(1-e^{-t / t_{0}}\right)$. In the limit $t_{0} \rightarrow 0$, this form approximates the step loading case. For fast loading (small $t_{0}$ ), the bridging traction law is initially stiff and then softens (see Fig.2). The crack opening displacement when all particle motion stops is greater than what it would have been under static loading to that stress level. We also see significant deviation from the static value even for moderate loading rates (especially from small time). Finally, the numerical scheme was also used to examine the influence of inertial effects on the traction law during a load-unload cycle. We consider loading the fiber instantaneously with a unit step load, wait for particle motion to cease and then remove the load instantaneously. The hysterisis behavior and the energy dissipated is significantly more when inertial effects are present (Figure 3). To conclude, inertial effects can significantly modify bridging behavior, even at moderate loading rates.

\section{CONCLUSIONS}

Analytical results have been presented for the problem of bridging by the mechanism of fibre pullout when the inertia of the fibre and the matrix are taken into account. We calculated simple criteria for significant inertial effects in the bridging mechanism in representative mode I crack propagation problems. We also find that for pullout or bridging stresses that rise linearly in time, the instantaneous crack displacement is less in the presence of inertial effects than it would be under static loading to the same bridging stress. However, solutions for pullout from a rigid matrix suggest that, if the bridging stress rises rapidly and is then held at a constant value, the crack displacement when all particle motion finally stops will be greater than it would have been under static loading to that stress level. Thus regimes of both hardening and softening of the bridging traction law due to inertial effects can be expected in bridged crack problems. We have also formulated numerical solutions for general loading cases and hence have a scheme to compute the bridging law for fairly general conditions. This will be required to solve large-scale bridging, dynamic crack problems to self-consistency.

ACKNOWLEDGMENTS: NS and BNC are grateful for support from the U.S. Army Research Office through contract number DAAD19-99-C-0042, administered by Dr. David Stepp, and IJB thanks the U.S. Dept. Of Energy for support through contract W-7405-ENG-36.

\section{REFERENCES:}

1. D. B. Marshall, B. N. Cox and A. G. Evans, Acta Metall. 33, 2013-2021 (1985).

2. $\quad$ L. N. McCartney, Proc. Roy. Lond. A409, 329-350 (1987).

3. J. W. Hutchinson and H. M. Jensen, Mechanics of Materials, 9, 139-163 (1990).

4. J. D. Achenbach, Wave Propagation in Elastic Solids, North-Holland, 1973, pp.138

5. B.N.Cox, N.Sridhar, and I.J.Beyerlein, submitted to J. Mech. and Phys. Solids, June 2000

6. L.V.Nikitin and A. N.Tyurekhodgaev, Wave Motion, 12, 513-526 (1990)

7. $\quad$ N.Sridhar, B.N.Cox and C.L.Dunn, in preparation for submission.

8. C.Hirsch, Num. Computation of Internal and External Flows, John Wiley,1988,p 408 